\title{
Modelling of Reduced Electric Field and Concentration Influence on Electron Transport Coefficients of $\mathrm{He}-\mathrm{Ne}$ Plasma
}

\author{
Maysam T. Al-Obaidi, Rafid A. Ali* and Baidaa Hamed \\ Department of Physics, College of Science, Mustansiriyah University, Baghdad, Iraq \\ Doi: 10.12693/APhysPolA.140.299 *e-mail: rafidphy_1972@uomustansiriyah.edu.iq
}

\begin{abstract}
A comprehensive theoretical investigation on the specific electron transport coefficient in the $\mathrm{He}-\mathrm{Ne}$ gas mixtures state in plasma has been carried out. The performed calculations enable us to measure the influence of the concentration of He:Ne gas mixture on plasma electronic coefficients. Our approach is based on the variation in the resistance of plasma field placed in a varying reduced electric field and thermodynamic equilibrium. The effects of reduced electric field and mixture concentrations on the electron mobility, diffusion coefficients, and total collision frequency have been calculated by solving the Boltzmann equation using the two-term approximation. The Monte Carlo simulation was used to solve the Boltzmann equation. The polynomial and logistic functions were adopted through the simulation process, and the appropriate equations have been provided, indicating the variation of plasma electronic coefficients to the variation of gas mixture concentration and reduced electric field. The applied reduced electric field has been chosen to be in the limited range of 0-100 Td, and is considered for several concentrations in the limited range of $0.1-0.7 \mathrm{~mol}$. The study shows that the obtained results are in good agreement with the results obtained using the Monte Carlo method.
\end{abstract}

topics: gas discharge plasma, electron transport coefficients, electron energy distribution function

\section{Introduction}

Plasma is defined as a "moldable" material [1] and is one among the four elemental states of matter, which was first expounded by chemist Irving Langmuir during the 1920s [2]. The term "plasma" is typically coined to explain a broad range of macroscopic neutral matters consisting of many closely interacting and colliding free electrons and ionized atoms or molecules, which show amalgamated behaviors and properties owing to the presence of long-range Coulomb energies and forces. It should be noted that the entire charged substances or particles field could not be brought under the classification of plasmas. There are some conditions or criteria to be satisfied to classify charged substances as plasma and to exhibit the plasma properties [3]. For instance, an ionized substance, which is macroscopically neutral with a characteristic length smaller than the Debye length could be considered as plasma. The primary interactions in plasma are electromagnetic interactions that essentially originated from the presence of an abundant number of charged substances/particles in plasma $[4,5]$.

Since plasma largely contains electrically conductive gaseous ionized substances, it enables the long-range electric and magnetic fields to control the behavior and properties of matters. The characteristics such as the existence of high conductivity and response to the electric/magnetic fields make plasma potentially useful in a wide variety of applications. Firstly, a suitable control is needed. Secondly, the potential energy sources or radiations are required to achieve the plasma. Therefore, the lasers are routinely harnessed to produce a variety of plasma types in the laboratory $[6,7]$.

Furthermore, the characteristic properties of plasma are remarkably dependent on the interactions among the substances in plasma. Notably, the presence of "collective effects" in plasma is often found to be one of the fundamental features that differentiate the plasma from that of the other typical solids and fluids. Furthermore, it is because of the existence of long-range electromagnetic forces. Every individual charged substance in the plasma simultaneously interacts with more number of other charged particles, manifesting the "collective effects" which are the key factors for the effective physical phenomena occurring in plasma $[8,9]$.

The precise collision cross-section and rate coefficient values are crucial for determining the kinetic processes through modeling [1-3, 8, 9], simulation [4] and diagnostics $[5,6,8,9]$ of the discharge processes $[10,11]$. 
It is known that the plasma at low temperature contains an adequate amount of neutral substances, where their greater internal degree of freedom enables their active collisions and interactions with electrons in the plasma. The existence of surplus electrons and other interaction channels, such as ionization/recombination reactions for heavy plasma substances, substantially leads to the reformulation of electron transport equations and mathematical expressions for various factors, such as electron diffusion, electrical conductivity, thermal conductivity, and viscosity coefficients. Thus, the role of inelastic collisions of electrons towards the deduction of electron transport coefficient has become the subject of interest $[12,13]$.

The electron transport processes in plasma and gaseous substances have been extensively investigated over the last few decades through theoretical and experimental studies. Most of the theoretical investigations were devoted primarily to the analysis of scalar transport features of electrons since the rate of chemical reactions and electron energy relaxations are crucial and constitute the focal point of research in plasma [14].

The fluid model-based gas discharge processes typically require the transport- and rate-coefficients as the input. The coefficients are typically estimated from the collision cross-section data by using the electron energy Boltzmann equation (EEBE) [15], and are largely associated with the electron energy distribution function (EEDF). In fact, the Maxwell-Boltzmann distribution functions which are obtained from the kinetic theory of gaseous substances provide a facile description for many basic gas properties including the properties such as diffusion, pressure, and velocity [16, 17].

In the present work, a mathematical model which is devoted to theoretical investigation on specific electron transport coefficient of $\mathrm{He}-\mathrm{Ne}$ plasma is predicted by solving Boltzmann equation utilizing the two-term approximation and by taking the advantages of Monte Carlo simulation method.

\section{Mathematical model}

In this work, the BOLSIG ${ }^{+}$program has been employed to solve the Boltzmann equation (BE) using the direct simulation Monte Carlo (DSMC) method which is based on the Monte Carlo (MC) simulation for the finite number fluid-flows [18]. $\mathrm{BOLSIG}^{+}$is considered a more generic and easierto-use tool as compared to other BE solving methods. A wide variety of fundamental processes in gas-discharging plasma can be assessed and estimated using BE which typically include the process of electron-atom collisions, elastic collisions, electron impacted atom excitation, the transition of collisions between the surrounded atoms, and the ionatom collisions resulting from the elastic collisions. In the present work, the electron mobility, diffusion coefficients, and total collision frequency have been calculated with varying reduced electric field $E / N$, several concentrations of helium-neon $(\mathrm{He}-\mathrm{Ne})$ gas mixture, above the excitation temperature of $300 \mathrm{~K}$.

Variations of mixture concentration can specify the He-Ne plasma electronic transport coefficients for many applications.

\subsection{The Boltzmann equation}

Boltzmann introduced the probability via expounding the statistical characteristics of a nonequilibrium thermodynamic system. Probability has also been used in quantum physics to describe the concept of time reversal, associating the direction of probability with the entropy exchange and breaking the similarity between the past and the future $[19,20]$ - even though the laws of mechanics remain unchanged for time inversion [21, 22].

The Boltzmann relation gives the ratio of number density (i.e., number per unit volume) of the substances such as atoms, ions or molecules at a certain energy level $N_{2}$ to the number density in lower energy level $N_{1}$. This can be written as [23]

$$
\frac{N_{2}}{N_{1}}=\frac{G_{2}}{G_{1}} \exp \left(-\frac{E}{k_{\mathrm{B}} T}\right),
$$

where $G_{1}$ and $G_{2}$ denote the multiplicity of two energy positions, $E$ is the energy required to excite the particles, $k_{\mathrm{B}}$ is the Boltzmann constant, and $T$ is the temperature. Notably, a greater number of particles can be excited by increasing $T[18,20]$.

The Boltzmann equation (BE) is

$$
\frac{\partial f}{\partial t}=\left(\frac{\partial f}{\partial t}\right)_{\text {force }}+\left(\frac{\partial y}{\partial x}\right)_{\text {diff }}+\left(\frac{\partial f}{\partial t}\right)_{\text {coll }},
$$

where the first term represents "force" and it can be influenced by the external forces acting on the particles. In turn, the second term "diff" indicates the particle diffusions. Finally, the term "coll" indicates the forces acting between the particles in collision $[24,25]$.

\subsection{Equations of force, diffusion and collision terms}

The force term and diffusion term corresponding to $(2)$ can be written, respectively, as

$$
\left(\frac{\partial f}{\partial t}\right)_{\text {force }}=-\boldsymbol{F}(r, t) \cdot\left(\frac{\partial f}{\partial \boldsymbol{p}}\right)
$$

and

$$
\left(\frac{\partial y}{\partial x}\right)_{\text {diff }}=-\frac{p}{m} \cdot \nabla f
$$

The equivalent form of (2) is then

$$
\frac{\partial f}{\partial t}=\frac{\boldsymbol{p}}{m} \cdot \nabla f+\boldsymbol{F}(\boldsymbol{r}, t) \cdot\left(\frac{\partial f}{\partial \boldsymbol{p}}\right)+\left(\frac{\partial f}{\partial t}\right)_{\text {coll }},
$$

Here, $\boldsymbol{F}(\boldsymbol{r}, t)$ represents the field exerting on the particles in fluid, $\boldsymbol{p}$ is the momentum, and $m$ is the particle mass. The terms on the right side of (5) describe the effects of particle collisions, where it will be zero if there is no collision between the particles [26]. Regarding the "collision" term, many 
efforts have been taken towards modelling the collision. In this direction, the models/equations proposed by Bhatnagar, Gross and Krook (known as BGK form) [26] is one of the well-known models/equations. The modified BE as per the BGK form is given as

$$
\frac{\partial f}{\partial t}+\frac{\boldsymbol{p}}{m} \cdot \nabla f+\boldsymbol{F} \cdot \frac{\partial f}{\partial \boldsymbol{p}}=\nu\left(f_{0}-f\right),
$$

where $\nu$ denotes the frequency of collision and $f_{0}$ is the local Maxwillian distribution function.

\section{General Boltzmann equation for a particle mixture}

For a chemical mixture of particles categorized by the indices $i=1,2,3, \ldots, n$, one has the following

$$
\frac{\partial f_{i}}{\partial t}+\frac{\boldsymbol{p}_{i}}{m_{i}} \cdot \nabla f_{i}+\boldsymbol{F}(\boldsymbol{r}, t) \cdot \frac{\partial f_{i}}{\partial \boldsymbol{p}_{i}}=\frac{\partial f_{i}}{\partial t}
$$

with $f_{i}=f_{i}\left(\boldsymbol{r}, \boldsymbol{p}_{i}, t\right)$ and where $\boldsymbol{r}$ represents the position of particle, $\boldsymbol{p}_{i}$ and $m_{i}$ are the momentum and mass of species, respectively. The collision term can be given by

$$
\begin{aligned}
& \left(\frac{\partial f_{i}}{\partial t}\right)_{\text {coll }}= \\
& \sum_{j=1}^{n} \iint \mathrm{d}^{3} \boldsymbol{p}^{\prime} \mathrm{d} \Omega g_{i j} I_{i j}\left(g_{i j} \Omega\right)\left(f_{i}^{\prime} f_{j}^{\prime}-f_{i} f_{j}\right) .
\end{aligned}
$$

It can be seen that the integration is over the components of momentum, where $f^{\prime}=f^{\prime}\left(\boldsymbol{p}_{i}^{\prime}, t\right)$, $I_{i j}$ represents the differential cross-sections between the species $i$ and $j$, and $\mathrm{d} \Omega$ denotes the solid angle of collisions. The relative momentum $g_{i j}$ can be given by

$$
g_{i j}=\left|\boldsymbol{p}_{i}-\boldsymbol{p}_{j}\right|=\left|\boldsymbol{p}_{i}^{\prime}-\boldsymbol{p}_{j}^{\prime}\right|,
$$

where $\boldsymbol{p}_{i}, \boldsymbol{p}_{j}$ and $\boldsymbol{p}_{i}^{\prime}, \boldsymbol{p}_{J}^{\prime}$ are the $i, j$-th momentums before and after collisions, respectively [27].

\section{Results and discussion}

Figure 1a and b represents the collisional crosssection area of $\mathrm{He}$ and $\mathrm{Ne}$. To solve the BE, one needs substantial information about the collisional cross-section of the gas mixture considered. Figure 1 shows the cross-section with respect to the electron energy of the reaction involved. Difference in atomic structure between $\mathrm{He}$ and Ne leads to different energy levels for each gas, and consequently, to theirs different behavior (as can be seen in Fig. 1a and $b$ ). The output data has been included in the current calculations.

Figure $2 \mathrm{a}$ and $\mathrm{b}$ shows that there is a significant dependence of the electron energy distribution function (EEDF) on the electron mean energy (for several values of applied electric field $E / N)$. The number of particles is $N=2.7 \times 10^{25} \mathrm{~m}^{-3}$. The obtained results also show that the electrons can be affected by a forward bias by increasing the applied electric field $E / N$. This bias will accelerate the electrons and increase energy up to a steady level of
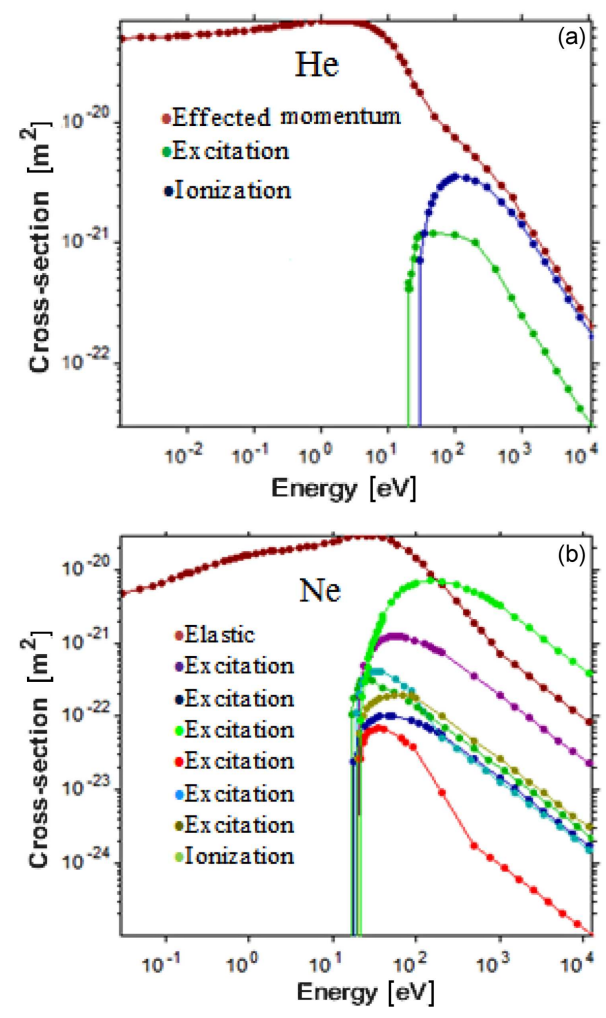

Fig. 1. The collisional cross-section of (a) He and (b) Ne as a function of electron energy.
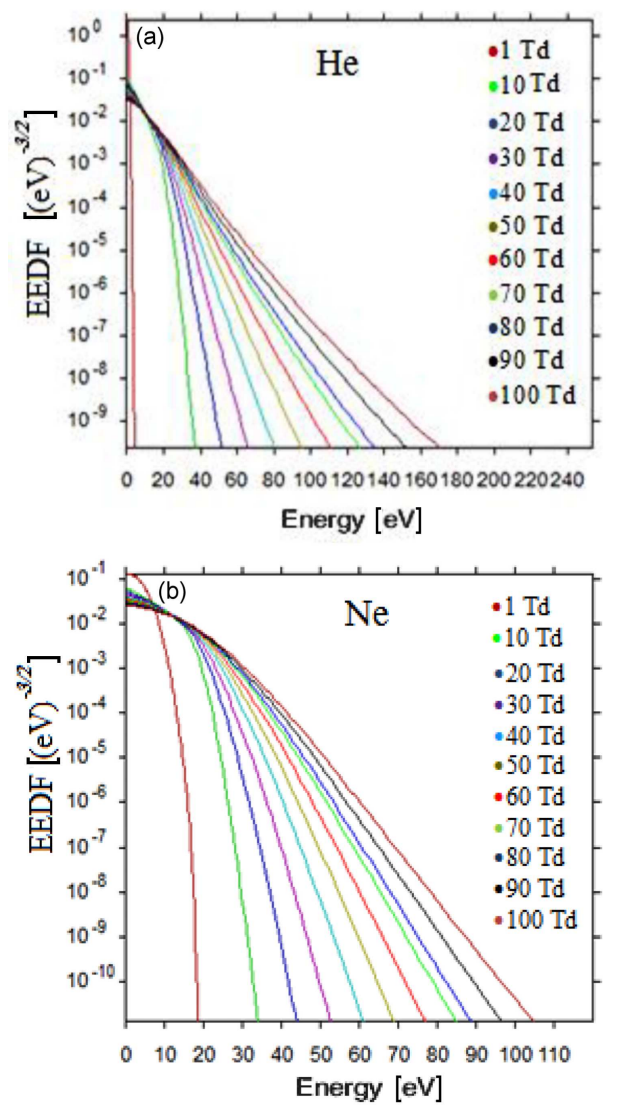

Fig. 2. EEDF of (a) He and (b) Ne as a function of the electron energy. 
mean energy, which eventually increases the rate of inelastic collisions. Above this point of the steady energy level, the increment in $E / N$ will be ineffective and the curve will fall towards the high-energy tail. In other words, the high values of EEDF will be at the low values of energy $u$. When increasing the $u$ values, the EEDF will approach the original point due to the high energy electron resulting from the applied forward bias. Values of EEDF for several concentrations of He-Ne mixture are quasi logarithmic according to the slope of $1 /\left(k_{\mathrm{B}} T\right)$. The shape of the curves, as displayed in Fig. 2, illustrates the Maxwell distribution for the several mixture concentrations.

\subsection{Influence of $E / N$ on the electron mobility}

In Fig. 3, the variation in electron mobility $\mu / N$ can be observed with respect to the increasing $E / N$ for several concentrations $(0.1-0.6 \mathrm{~mol})$ of $\mathrm{He}-\mathrm{Ne}$ mixtures, where the sharp decrease of $\mu / N$ occurs between 0 and $40 \mathrm{Td}$. In the further range of $E / N$, i.e., 40-100 Td, the mobility values become almost steady. This can be caused by the descending electron energy as a result of ionization and excitation processes [28].

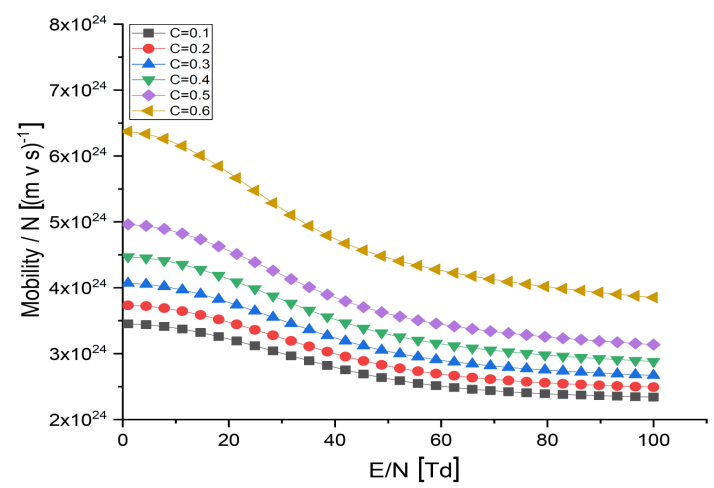

Fig. 3. Mobility as a function of different concentrations.

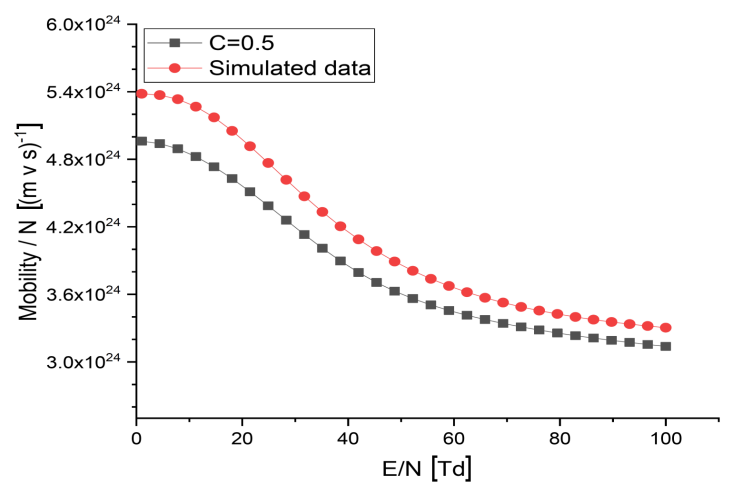

Fig. 4. Change in energy mobility with respect to $E / N$ (obtained by the BOLSIG $^{+}$and the estimated data).

\subsection{Influence of the mixture concentration on the mobility}

In Table I, the influence of mixture concentration on electrons mobility can be observed by analyzing the $C$ behavior from a low $0.1 \mathrm{~mol}$ to its higher value $0.7 \mathrm{~mol}$. The observed effect results from the excitation and ionization processes in relation to the reduced electric field $E / N$.

\subsection{Modeling of the mobility}

We have calculated the concentration values using the $\mathrm{BOLSIG}^{+}$program which bases on the description of the mobility behavior $(\mu / N)$ depending on $E / N$. The obtained results are presented in Fig. 4. Notably, the present $\mathrm{BOLSIG}^{+}$data are found to be well-matched with the data obtained in our present work (see Fig. 4).

The fitting relationships for several concentrations can be written as

$$
\frac{\mu}{N}=A_{2}+\frac{A_{1}-A_{2}}{1+\left(\frac{E / N}{X_{0}}\right)^{P}},
$$

where $E / N=0-100 \mathrm{Td}$, while $A_{1}, A_{2}, X_{0}, P$, for a given concentration value, are constants. Polynomial form has been assumed for

$$
\begin{aligned}
& A_{1}=\left(3.32+0.9 C+4.87 C^{2}\right) \times 10^{24}, \\
& A_{2}=\left(2.91+0.46 C+2.34 C^{2}\right) \times 10^{24}, \\
& X_{0}=36.6+7.8 C-16.22 C^{2}, \\
& P=2.65-0.6 C+0.1 C^{2},
\end{aligned}
$$

to determined the required concentration $C$.

\subsection{Influence of $E / N$ on the diffusion coefficients $D N$}

Figure 5 shows the variation in diffusion coefficient $(D N)$ with respect to the variation in the electric field $E / N$. A slight/small increment in $D N$ is observed in the limited range of $0<E / N<20 \mathrm{Td}$, while a clear $D N$ increment is observed in the range of $20 \mathrm{Td}<E / N=20-60 \mathrm{Td}$. Next, in the range of 60-100 Td, the $D N$ values increase slightly.

TABLE I

Influence of the mixture concentration $C$ on the electron mobility $\mu / N(E / N=55.62 \mathrm{Td})$.

\begin{tabular}{c|c}
\hline \hline Concentration $C[\mathrm{~mol}]$ & $\mu / N \times 10^{25}[1 /(\mathrm{m} \mathrm{V} \mathrm{s})]$ \\
\hline 0.1 & 0.254 \\
0.2 & 0.237 \\
0.3 & 0.287 \\
0.4 & 0.320 \\
0.5 & 0.350 \\
0.6 & 0.387 \\
0.7 & 0.433
\end{tabular}




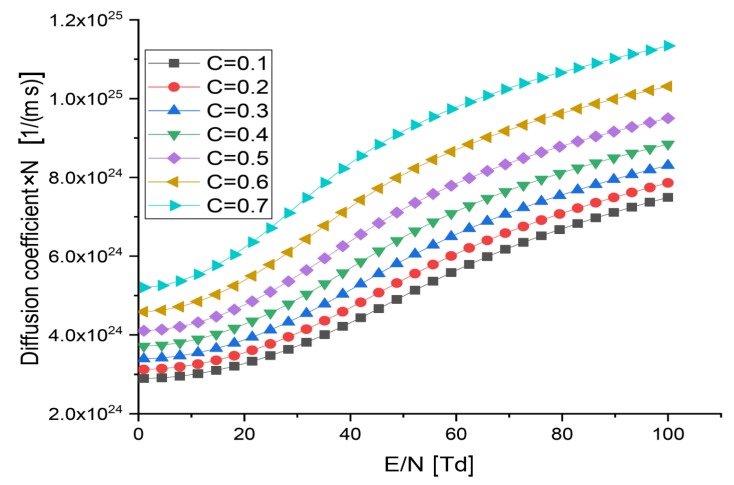

Fig. 5. Diffusion coefficient as a function of $E / N$ at different concentration values $C$.

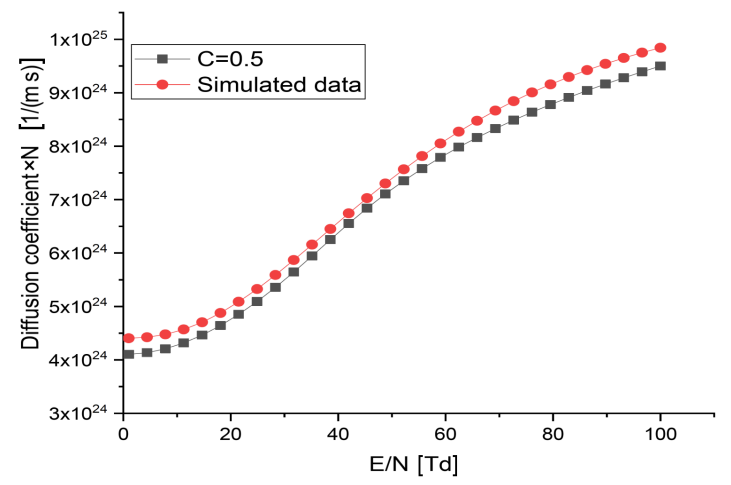

Fig. 6. Diffusion coefficient as a function of $E / N$ (obtained by BOLSIG ${ }^{+}$and our estimated data.

TABLE II

Influence of mixture concentration $C$ on diffusion coefficient $D N(E / N=41.97 \mathrm{Td})$.

\begin{tabular}{c|c}
\hline \hline Concentration $C[\mathrm{~mol}]$ & $D N \times 10^{25}[1 /(\mathrm{m} \mathrm{s})]$ \\
\hline 0.1 & 0.44 \\
0.2 & 0.48 \\
0.3 & 0.53 \\
0.4 & 0.58 \\
0.5 & 0.65 \\
0.6 & 0.74 \\
0.7 & 0.85
\end{tabular}

The growth of $D N$ is essentially due to the electronion and electron- $\mathrm{n}$ collisions, which caused the drift of the mass centre of atom (ion) and a subsequent rerelease of electrons.

\subsection{Influence of the concentration on $D N$}

Table II shows the variation in the diffusion coefficient $D N$ with respect to the variation of mixture concentration $C$ for a specific value of reduced electric field $E / N=41.97 \mathrm{Td}$. This direct relationship between $D N$ and $C$ is due to the inelastic collisions.

\subsection{Diffusion coefficients modeling}

The behavior of $D N$ with respect to the change in $E / N$ is shown in Fig. 6. The estimated points are obtained using the logistic function of the form

$$
D N=B_{2}+\frac{B_{1}-B_{2}}{1+\left(\frac{E / N}{r_{0}}\right)^{r}},
$$

where $E / N=0-100 \mathrm{Td}$, and $B_{1}, B_{2}, r_{0}, r$ are constants for a given values of $C$. Therefore, the fitting for all concentrations is reflected with (15).

The parameters $B_{1}, B_{2}, r_{0}, r$ have been obtained utilizing polynomial function, respectively, as

$$
\begin{aligned}
& B_{1}=\left(2.81+0.892 C+0.356 C^{2}\right) \times 10^{24}, \\
& B_{2}=\left(9.5-0.615 C+6.9 C^{2}\right) \times 10^{24}, \\
& r_{0}=73.3-34.16 C-6.55 C^{2}, \\
& r=2.36-0.073 C-0.3 C^{2} .
\end{aligned}
$$

Therefore, the concentration required in (15) is determined with the use of (16)-(19).

Figure 6 shows a well-matched result between the data obtained by the $\mathrm{BOLSIG}^{+}$program and the estimated data with (15) for a specific concentration value $C=0.5 \mathrm{~mol}$.

\subsection{Influence of $E / N$ on total collision frequency}

Figure 7 shows the dependence of total collision frequency (TCF) on $E / N$ of the $\mathrm{He}-\mathrm{Ne}$ mixture. In the range of $E / N<20 \mathrm{Td}$, a slight increment in the TCF is observed, whereas a sharp TCF increment is observed for $E / N>20 \mathrm{Td}$. This result is referred to the fact that the electric energy and cross-section depend on the electron excitation and ionization energy [28].

Table III shows the values of TCF of the $\mathrm{He}-\mathrm{Ne}$ mixture for a specific value of the reduced electric field. High TCF values are observed at a low mixture concentration of $0.1 \mathrm{~mol}$ compared to a high mixture concentration of $0.7 \mathrm{~mol}$. This can be ascribed to the high inelastic collisions rate and the collisional cross-section at low concentrations.

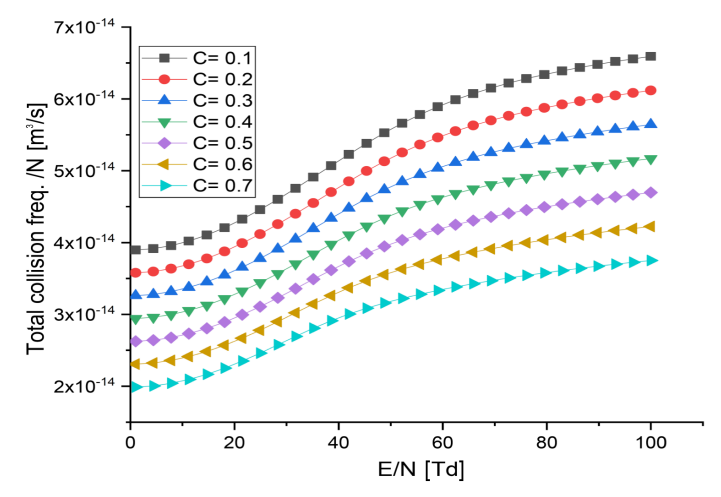

Fig. 7. Total collision frequency as a function of $E / N$ at different concentrations $C$. 


\subsection{Total collision frequency (TCF) modeling}

The behavior of TCF with respect to the varying values of $E / N$ is shown in Fig. 8. The estimated points of the total collision frequency $\nu_{\mathrm{eo}} \mathrm{T}$ are obtained using the logistic function of the form

$$
\frac{\nu_{\mathrm{eoT}}}{N}=G_{2}+\frac{G_{1}-G_{2}}{1+\left(\frac{E / N}{n_{0}}\right)^{n}},
$$

where $E / N=(0-100) \mathrm{Td}$.

The parameters $G_{1}, G_{2}, n_{0}, n$ which are constants for a given value of $C$, are obtained using polynomial function, respectively,

$$
G_{1}=\left(4.25-3.22 C-8.38 \times 10^{-4} C^{2}\right) \times 10^{-14},
$$

$$
\begin{aligned}
& G_{2}=\left(7.56-5.2 C+0.176 C^{2}\right) \times 10^{-14}, \\
& n_{0}=48.03-2.68 C-7.28 C^{2}, \\
& n=2.47-0.039 C-0.486 C^{2} .
\end{aligned}
$$

Therefore, the concentration $C$ required in (20) is determined with the use of $(21)-(24)$.

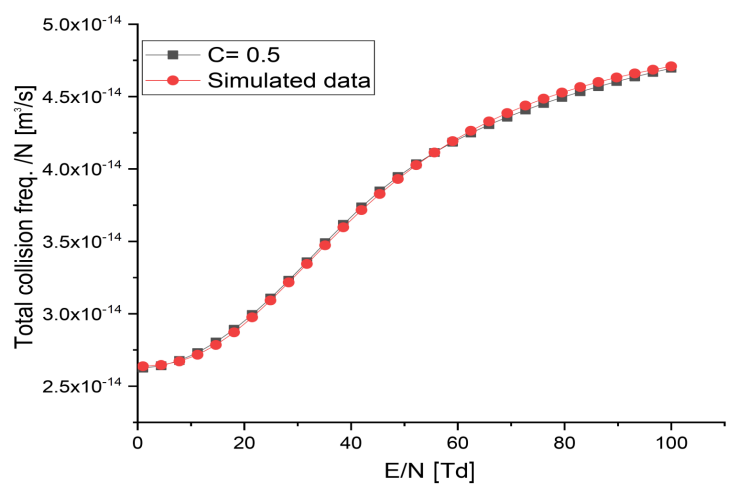

Fig. 8. Total collision frequency results show wellmatching between the BOLSIG ${ }^{+}$and the estimated data under specific concentration $C$ of $0.5 \mathrm{~mol}$.

TABLE III

Total collision frequency $\nu_{\text {eot }}$ in the $\mathrm{He}-\mathrm{Ne}$ mixture under the effect of the reduced electric field $(E / N=525.6 \mathrm{Td})$.

\begin{tabular}{c|c}
\hline \hline Concentration $[\mathrm{mol}]$ & $\nu_{\mathrm{eoT}} / N \times 10^{-13}\left[\mathrm{~m}^{3} / \mathrm{s}\right]$ \\
\hline 0.1 & 0.578 \\
0.2 & 0.536 \\
0.3 & 0.494 \\
0.4 & 0.453 \\
0.5 & 0.411 \\
0.6 & 0.369 \\
0.7 & 0.328
\end{tabular}

\section{Conclusions}

The effect of induced electric field and plasma state in the He-Ne gas mixture concentrations on the electron energy distribution function (EEDF) has been investigated. The results are obtained with the Boltzmann equation by the $\mathrm{BOLSIG}^{+}$program supported by the direct simulation Monte Carlo method. It is observed that increasing the electric field and the concentration of mixture increase the EEDF due to inelastic collisions which influence the behavior of EEDF and are closely related to the electronic coefficients. The changes observed in EEDF with respect to the electric field indicated that the performed calculations and results obtained agree with the Maxwell equations.

The logistic and polynomial functions were fitted to the data. We have obtained the appropriate and simplified equations to observe the behavior of transport electronic coefficients. The results also showed that the data from the BOLSIG ${ }^{+}$ Monte Carlo method were found to be well-matched with the estimates obtained in the fitting process performed in this study. Also, the output results turned out to be well suited to other studies [10, 27]. Overall, the obtained results suggested that the Boltzmann equation (BE) can be one of the most perspective tools to investigate the plasma states and their electronic coefficients from the kinetics of electrons in feebly ionized gaseous substances.

\section{Acknowledgments}

Authors would like to express their thanks and gratitude to the Department of physics, College of Science, Mustansiriyah University for support and cooperation.

\section{References}

[1] M.C. Kelley, The Earth's Ionosphere: Plasma Physics and Electrodynamics, 2nd ed., Academic Press, 2012.

[2] A.I. Morozov, Introduction to Plasma Dynamics, 1st ed., CRC Press, 2012, p. 17.

[3] J.A. Bittencourt, Fundamentals of Plasma Physics, 3rd ed., Ch. 1, Springer, New York 2004.

[4] F.L. Braga, Appl. Math. Lett. 24, 653 (2011).

[5] O.S. Vaulina, E.A. Linsin, E.A. Sametov, R.A. Timirkhanov, Plasma Fusion Res. 13, 1406125 (2018).

[6] P.K. Chu, X.P. Lu, Low Temperature Plasma Technology: Methods and Applications, CRC Press, 2020.

[7] P.J. Freidberg, Plasma Physics and Fusion Energy, Cambridge University Press, 2007, p. 121. 
[8] S.N. Antipov, M.M. Vasilev, S.A. Maiarov, O.F. Petrov, V. E. Fortov, J. Exp. Theor. Phys. 112, 482 (2011).

[9] A. Piel, Plasma Physics: An Introduction to Laboratory, Space, and Fusion Plasmas, 2nd ed., Springer, 2010, p. 4.

[10] V.S. Kurbanishmailov, S.A. Maiorov, O.A. Omarov, G. Ragimkhanov, I.G. Ramazanov, Bull. Dagestan State Univ., Series 1, Nat. Sci. 33, 7 (2018) (in Russian).

[11] I.R. Golyatina, A.S. Maiorov, Bull. Lebedev Phys. Inst. 41, 285 (2014).

[12] Z.W. Cheng, X.M. Zhu, F.X. Liu, Y.K. Pu, J. Plasma Sources Sci. Technol. 24, 025025 (2015).

[13] E.A. Jawad, Ibn Al-Haitham J. Pure Appl. Sci. 30, 38 (2017).

[14] V.M. Zhdanov, A.A. Stepanenko, Phys. Proced. 71, 110 (2015).

[15] A. Vikharev, A. Gorbachev, D. Radishev, J. Phys. D Appl. Phys. 52, 014001 (2018).

[16] M. Yitzhak, Phys. Plasmas 27, 060901 (2020).

[17] C. Köhn, O. Chanrion, T. Neubert, Plasma Sources Sci. Technol. 26, 015006 (2017).
[18] Y. Lin, F. Wang, B. Liu, J. Comput. Phys. 360, 93 (2018).

[19] B. Evans, S.P. Walton, Appl. Math. Model. 52, 215 (2017).

[20] M. Drewes, C. Weniger, S. Mendizabal, Phys. Lett. B. 718, 1119 (2013).

[21] S. Yatom, A. Shlapakovski, L. Beilin, E. Stambulchik, S. Tskhai, Y.E. Krasik, Plasma Sources Sci. Technol. 25, 064001 (2016).

[22] P.T. Gressman, R.M. Strain, Proc. Natl. Acad. Sci. 107, 57445749 (2010).

[23] R.I. Golyatinaa, S.A. Maiorova, Plasma Phys. Rep. 44, 453 (2018).

[24] G.I. Sukhinin, M.V. Salnikov, AIP Conf. Proc. 1925, 020029 (2018).

[25] P.T. Gressman, R.M. Strain, J. Am. Math. Soc. 24, 771 (2011).

[26] D.A. Storozhev, S.T. Surzhikov, Teplofizika vysokih temperatur 53, 307 (2015) (in Russian).

[27] I.Y. Bychkov, S.A. Yampolskaya, A.G. Yastremskii, Plasma Phys. Rep. 39, 374 (2013).

[28] W. Yang, X. Meng, X. Zhou, Q. Zhou, Z. Dong, AIP Adv. 9, 035041 (2019). 